\title{
Impact des pratiques de gestion des adventices sur le rendement du riz au centre de la Côte d'Ivoire
}

\author{
N.K.M. BORAUD ${ }^{1,2^{*}}$, Kra F. KOUAME ${ }^{1,2}$ et D. KLA ${ }^{1}$ \\ ${ }^{1}$ Laboratoire de Botanique, U.F.R. Biosciences, Université Félix Houphouët Boigny, Côte d'Ivoire. \\ ${ }^{2}$ Centre National de Floristique (C.N.F.) Université F.H.B., Laboratoire de Botanique, UFR Biosciences 22 \\ BP. 582 Abidjan 22, Côte d'Ivoire. \\ *Auteur correspondant, E-mail: boraudn@hotmail.com
}

\section{RESUME}

L'objectif de cette étude était de déterminer et d'évaluer l'impact des pratiques paysannes de gestion de l'enherbement sur la production rizicole en Côte d'Ivoire. Une enquête et la technique des relevés floristiques basée sur un «tour du champ» ont été utilisées. Les relevés floristiques ont permis de recenser 16 espèces végétales réparties en 14 genres appartenant à 10 familles botaniques sur l'ensemble des zones d'étude. Il existe une homogénéité floristique du milieu d'étude. L'enquête réalisée auprès des producteurs révèle une influence des pratiques culturales sur la production rizicole. La riziculture irriguée pratiquée à Bounguè offre un meilleur rendement que celle de la riziculture inondée à Raviart.

(C) 2015 International Formulae Group. All rights reserved.

Mots clés: Relevés, floristique, riziculture, rendement.

\section{INTRODUCTION}

Le riz constitue l'aliment de base de la population mondiale. Il représente une denrée alimentaire incontournable pour l'Afrique occidentale. Celle-ci importe annuellement 5,2 millions de tonnes de riz (FAO, 2008). Malgré son importance dans l'alimentation mondiale, la riziculture est la $4{ }^{\text {ème }}$ spéculation en Côte d'Ivoire parmi les principales cultures après l'igname, le manioc et la banane plantain. La riziculture pluviale représente 97 $\%$ des superficies rizicoles. Seuls $3 \%$ des sols rizicoles bénéficient d'une irrigation totale ou partielle (Bouet et al., 2005); Djato et al., 2006 ; Sangaré et al., 2009). Selon le Centre National de Recherche Agronomique
(CNRA), la production annuelle ivoirienne est de 600000 tonnes de riz blanchi pour des besoins estimés à 1500000 tonnes. Le riz est la première céréale consommée en Côte d'ivoire (60 kg/hab/an).

Le déficit de production du riz peut être expliqué par un environnement socioéconomique défavorable. Les contraintes de production en riziculture pluviale sont d'ordre : climatique (rareté, irrégularité et insuffisance des pluies) ; parasitaire (prévalence de pyriculariose et l'helminthosporiose); édaphique (baisse de la fertilité chimique des sols) ; variétal (cultivars peu productifs). Concernant la riziculture irriguée les principales contraintes sont la non maîtrise de 
l'eau d'irrigation, la panachure jaune (RYMV), la pyriculariose et la toxicité ferreuse. Cependant, chez ces deux types de rizicultures, le rendement est fortement influencé par l'envahissement des parcelles cultivées par les mauvaises herbes. La baisse de rendement subséquente à ce phénomène varie entre 9 et $32 \%$ dans les rizières du monde (Oerke et Dehne, 2004). La nature de leur agressivité est fonction des pratiques de gestion et des facteurs biophysiques. Les mauvaises herbes entraînent des pertes de rendement estimées à au moins 2,2 millions de tonnes par an en Afrique sub-saharienne (Rodenburg et Johnson, 2009). Dans la mesure d'une possibilité de lutte, quelles sont les méthodes envisageables ? Pour répondre à ces préoccupations, une étude intitulée «Impact des pratiques de gestion des mauvaises herbes (adventices) sur le rendement du riz au Centre de la Côte d'Ivoire» a été entreprise.

L'objectif de cette étude a été de déterminer et d'évaluer l'impact des pratiques paysannes de gestion de l'enherbement sur la production rizicole en Côte d'Ivoire.

\section{MATERIEL ET METHODES}

\section{Milieux d'étude}

Les études se sont déroulées dans deux localités Boungué et Raviart situés dans le département de Didiévi de la région du Bélier au centre de la Côte d'ivoire.

Le périmètre rizicole aménagé de Bounguè est situé dans le domaine guinéen et dans le secteur pré forestier au centre de la Côte d'Ivoire. Il se situe entre les coordonnées géographiques suivantes : $7^{\circ} 09^{\prime}$ à $7^{\circ} 24^{\prime}$ de latitude Nord et $4^{\circ} 53^{\prime}$ à $5^{\circ} 29^{\prime}$ de longitude Ouest. Sur le plan administratif, il fait partie de la sous-préfecture de Molonou-Blé.

Le périmètre rizicole non aménagé de Raviart est situé dans le domaine guinéen et dans le secteur pré forestier. Il est situé dans la vallée du KAN près du chef-lieu de la sous- préfecture de Raviart qui et a comme coordonnées géographiques : $7^{\circ} 24^{\prime}$ de latitude Nord et $4^{\circ} 53$ de longitude Ouest.

Le département de Didiévi auquel appartient Bounguè et Raviart dispose des sols profonds, de couleur rouge ocre, à texture argilo-sableuse, gravillonnaire et latéritiques à la base. Ce sont essentiellement des ferralsols (Akado et Djidji, 1990). Les sols se distinguent au niveau des plateaux avec des brunisol et des fluvisols dans les plaines et les bas-fonds.

\section{Matériel biologique}

Le matériel biologique de cette étude est constitué par :

- les différentes variétés de riz (WITA 4, WITA9, BOUAKE 184),

- $\quad$ les variétés de riz traditionnelles non sélectionnées,

- les mauvaises herbes ou adventices.

\section{Matériel technique}

Le matériel technique est constitué :

- d'un GPS (Global Positioning System) qui a permis de mesurer les superficies exploitées ;

- une charrue tractée par un zébu a été utilisée pour le labour ;

- de l'engrais (NPK et l'urée) et des herbicides ont été utilisés pour la fertilisation et le désherbage.

Les informations sur le terrain ont été recueillies sur des fiches d'enquêtes. L'outil informatique a permis de faire le traitement de texte sur Word. La saisie et l'exploitation des données ont été réalisées à l'aide du tableur EXCEL. Le logiciel STATISTICA 7.1 a permis de faire les tests U de Mann-Whitney et l'analyse de variance (Anova 1).

\section{Méthodes}

\section{Approche méthodologique de l'enquête}

Ce travail a consisté à identifier les pratiques innovantes des riziculteurs en matière de gestion des adventices et d'évaluer 
dans quelles mesures elles sont généralisables et applicables. Il ne s'agit pas de caractériser des pratiques par des mesures précises, mais d'essayer d'avoir une vision globale de la gestion des adventices au sein des exploitations enquêtées. Pour atteindre nos objectifs, nous avons enquêté en interrogeant les riziculteurs sur:

- le type de labour,

- le type d'exploitation,

- la gestion des adventices

- le rendement.

\section{Technique des relevés floristiques}

Les relevés floristiques ont été réalisés selon un «tour du champ» qui consiste à parcourir la parcelle dans différentes directions à la recherche de nouvelles espèces non encore répertoriées. La superficie élémentaire de base utilisée est de 0,25 ha, correspondant à l'aire minimale au préalable identifiée dans des études similaires dans la zone d'étude (Kouamé, 2014). Dix (10) relevés reparties en fonction du type de culture du riz dont cinq (5) en culture de riz irrigué et cinq (5) en culture de riz pluvial sont effectués par localité.

\section{Analyse des données floristiques}

L'homogénéité floristique entre les deux localités est estimée par la détermination du coefficient de similitude selon la formule de Sørensen (1948)

$$
\text { Cs }=\stackrel{\text { 2.c }}{a+-----x} 100
$$

a et b représentent les listes des espèces recensées dans les unités d'échantillonnage que l'on veut comparer c est le nombre d'espèces communes aux deux listes $\mathbf{a}$ et $\mathbf{b}$. D'après cette formule, la valeur du coefficient de similitude (Cs), exprimée en pourcentage, varie entre 0 et $100 \%$, selon que les deux listes ont des compositions floristiques totalement différentes (dans ce cas, elles ne comportent aucune espèce commune et $\mathrm{c}=0$ ) ou, selon que les deux listes sont rigoureusement identiques ; dans ce cas, $a=b=$ c. Lorsque le coefficient de similitude est supérieur ou égal à $50 \%$, cela signifie que les deux milieux sont considérés comme étant floristiquement homogènes (Boraud, 2010).

\section{Analyse des données d'enquêtes}

Les données recueillies sont analysées grâce au tableur EXCEL. La gestion des données s'articule autour de deux points essentiels.

Le premier point consiste à regrouper la population selon les différentes pratiques culturales (le mode de labour et le type de désherbage).

Le second point consiste à calculer les rendements moyens en fonction des types d'exploitations.

Le rendement individuel est le rapport de la production de la parcelle (t) sur la surface cultivée (Kouamé, 2014).

Le rendement moyen est le rapport de la somme des rendements individuels sur le nombre de personnes interrogées $(\mathrm{N})$ :

$$
\mathrm{Qm}(\mathrm{t} / \text { personne })=\frac{\sum \text { Rendement individuel }(\mathrm{Rm})}{\mathrm{N}}
$$

Avec Qm - Rendement moyen; N - Nombre de personnes enquêtées.

\section{Analyse statistique}

Pour déterminer l'impact des types de variétés sur le rendement du riz, le test $\mathrm{U}$ de Mann-Whitney équivalent du test de $\chi^{2}$ est effectué. Dans la présente étude où nous avons souvent comparé des moyennes de variables testées sur plus de deux groupes, nous avons utilisé l'analyse de variance à un facteur (ANOVA 1). Cette analyse est faite à l'aide du logiciel Statistica (version 7.1). Le 
niveau de significativité choisi pour ces analyses est de $5 \%(p=0,05)$. Lorsque la différence est significative, un test HSD de Tukey est réalisé pour classer les groupes homogènes. Le test d'homogénéité de la variance est réalisé par le test de Bartlett pour vérifier les conditions à l'application de l'analyse de variance (Benzecri, 1964, 1973 ; Fenelon, 1999).

\section{RESULTATS}

\section{Caractéristiques de la flore adventice}

Les relevés floristiques effectués ont permis d'identifier 16 espèces reparties en 14 genres appartenant à 10 familles (Tableau 1). Les monocotylédones représentent $43,75 \%$. Les Poaceae et des Cyperaceae se distinguent chacune respectivement avec $25 \%$ et $18,75 \%$. Il y a plus d'adventices dans les périmètres rizicoles de Raviart avec 15 espèces appartenant à 13 genres répartis en 9 familles. $\mathrm{Ce}$ sont: Ageratum conyzoides, Azolla africana, Chromolaena odorata, Cleome rutidosperma, Cyperus difformis, Cyperus haspan, Cyperus iria, Echinochloa crus-galli, Euphorbia heterophylla, Ludwigia hyssopifolia, Nymphaea lotus, Oryza sativa, Panicum repens, Physalis angulata et Rottboellia cochinchinensis.

A Bounguè, il y a 8 espèces reparties en 6 genres et appartenant à 5 familles botaniques. Ces espèces sont: Azolla africana, Cyperus difformis, Cyperus haspan, Cyperus iria, Commelina benghalensis, Echinochloa crus-galli, Oryza sativa et Euphorbia heterophylla. Cette richesse floristique a été établie sans tenir compte de la fréquence absolue des espèces inventoriées (Tableau 2).

\section{Homogénéité floristique}

Selon le Tableau 3, les localités de Bounguè et Raviart sont floristiquement homogènes avec un coefficient de similitude de $60,87 \%$. Ce qui signifie qu'il existe un cortège floristique de base à la riziculture des deux localités très proches géographiquement.

\section{Impact du type de gestion sur le rendement Impact de la localité et du type de labour}

L'enquête réalisée auprès des paysans révèle que le rendement moyen obtenu dans la riziculture irriguée chez les producteurs de Bounguè est de 4,7 t/ha contre 2,4 t/ha dans riziculture inondée du bas-fond chez les producteurs de Raviart (Tableau 4). L'analyse du test $\mathrm{U}$ de Mann-Whitney (test $U=151,00$; $p<0,005)$ a révélé qu'il y a une différence significative entre les rendements moyens obtenus dans chacune des localités (Figure 1).

Les analyses statistiques montrent que le rendement moyen obtenu avec le labour motorisé réalisé avec une bineuse de motoculteur est plus élevé que le labour manuel, le labour à traction animale et le non labour. Le rendement moyen obtenu en labour manuel (labour réalisé avec la daba) est estimé à $2,4 \mathrm{t} / \mathrm{ha}$ alors que celui du labour motorisé est de 4,0 t/ha et 2,2 t/ha pour la traction animale contre 1,6 t/ha pour le non labour. Trois classes de rendements sont distinguées en fonction du type de labour. La classe ab regroupe les rendements obtenus par le labour manuel et le labour à traction animale. La classe a pour les rendements du labour motorisé et la classe b pour le non labour.

\section{Impact du type de désherbage sur le rendement}

Il y a une variation du rendement en fonction du type de désherbage (Tableau 4).

Dans la localité de Bounguè, les producteurs pratiquent la riziculture irriguée avec un rendement moyen de 4 t/ha alors qu'à Raviart, nous avons la riziculture inondée avec un rendement de 2,7 t/ha. Dans le même ordre d'idée, le test $\mathrm{U}(U=190 ; p=0,001)$ et le tableau révèlent que le rendement obtenu au désherbage chimique est $4,7 \mathrm{t} / \mathrm{ha}$ contre $2 \mathrm{t} / \mathrm{ha}$ pour le désherbage manuel (Figure 3). 
Tableau 1 : Répartition des effectifs en familles, genres et espèces par localités.

\begin{tabular}{lcccc}
\hline & \multicolumn{2}{c}{ Raviart } & \multicolumn{2}{c}{ Boungue } \\
\cline { 2 - 5 } Familles & Genres & Espèces & Genres & Espèces \\
\hline Asteraceae & 2 & 2 & 0 & 0 \\
Azollaceae & 1 & 1 & 1 & 1 \\
Capparaceae & 1 & 1 & 0 & 0 \\
Cyperaceae & 1 & 3 & 1 & 3 \\
Commelinaceae & 0 & 0 & 1 & 1 \\
Euphorbiaceae & 1 & 1 & 1 & 1 \\
Nymphaeaceae & 1 & 1 & 0 & 0 \\
Onagraceae & 1 & 1 & 0 & 0 \\
Poaceae & 4 & 4 & 2 & 2 \\
Solanaceae & 1 & 1 & 0 & 0 \\
\hline Total & $\mathbf{1 3}$ & $\mathbf{1 5}$ & $\mathbf{6}$ & $\mathbf{8}$ \\
\hline
\end{tabular}

Tableau 2 : Répartition des espèces en fonction des localités.

\begin{tabular}{lccc}
\hline \multicolumn{1}{c}{ Familles } & Espèces & RAVIART & BOUNGUE \\
\hline Asteraceae & Ageratum conyzoides & + & - \\
Azollaceae & Chromolaena odorata & + & - \\
Capparaceae & Azolla africana & + & + \\
& Cleome rutidosperma & + & - \\
Cyperaceae & Cyperus difformis & + & + \\
& Cyperus haspan & + & + \\
Commelinaceae & Cyperus iria & + & + \\
Euphorbiaceae & Commelina benghalensis & - & + \\
Nymphaeaceae & Euphorbia heterophylla & + & + \\
Onagraceae & Nymphaea lotus & + & - \\
& Ludwigia hyssopifolia & + & - \\
Poaceae & Echinochloa crus-galli & + & + \\
& Oryza sativa & + & + \\
Solanaceae & Panicum repens & + & - \\
\hline
\end{tabular}

(-) : Absence ; (+) : Présence.

Tableau 3 : Coefficient de similitude des deux localités.

\begin{tabular}{|c|c|c|c|c|}
\hline Couples de localités & \multicolumn{3}{|c|}{ Nombre d'espèces } & \\
\hline Localités & $\mathrm{a}$ & $\mathrm{b}$ & $\mathrm{c}$ & Cs \\
\hline Raviart et Bounguè & 15 & 8 & 7 & 60,87 \\
\hline
\end{tabular}

Tableau 4 : Impact de la localité et du labour sur le rendement.

\begin{tabular}{lccc}
\hline \multicolumn{1}{c}{ Type de culture } & Rendement moyen $(\mathbf{t} / \mathbf{h a})$ & Type de labour \\
\hline Riziculture irriguée & 4,7 & 4 & Motorisé \\
& - & 2,4 & Traction animale \\
\hline Riziculture inondée & 2,4 & 2,2 & Manuel \\
& & 1,6 & Non labour \\
\hline
\end{tabular}

$U=151,00 ; p=0,0001)$ 


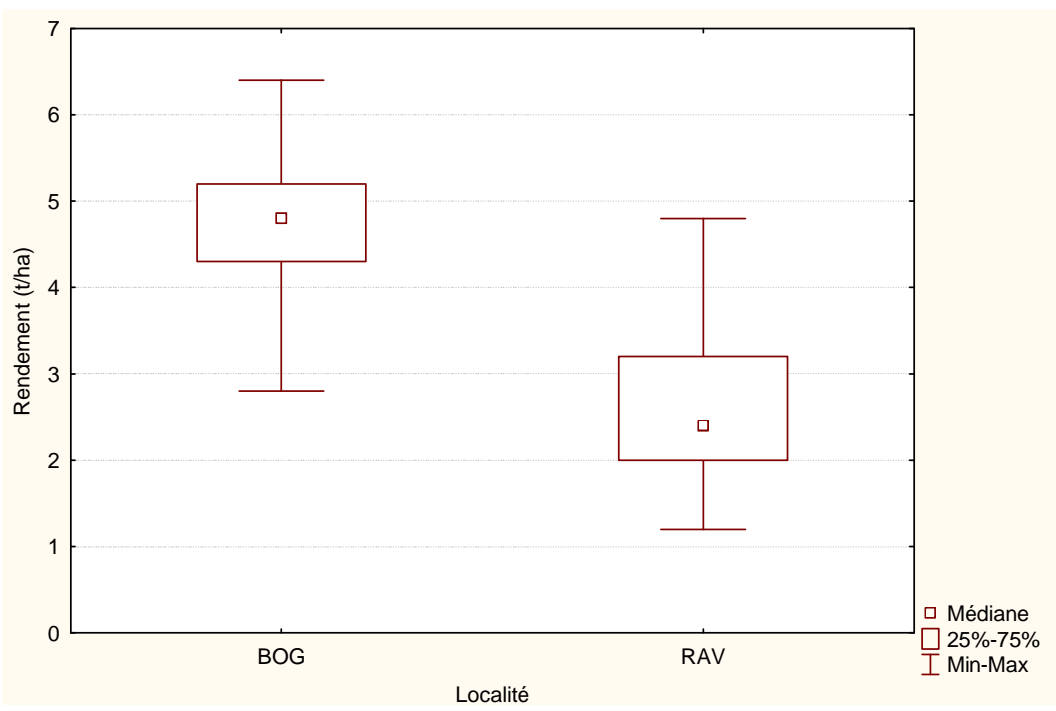

Figure 1 : Comparaison des rendements moyens en fonction des localités.

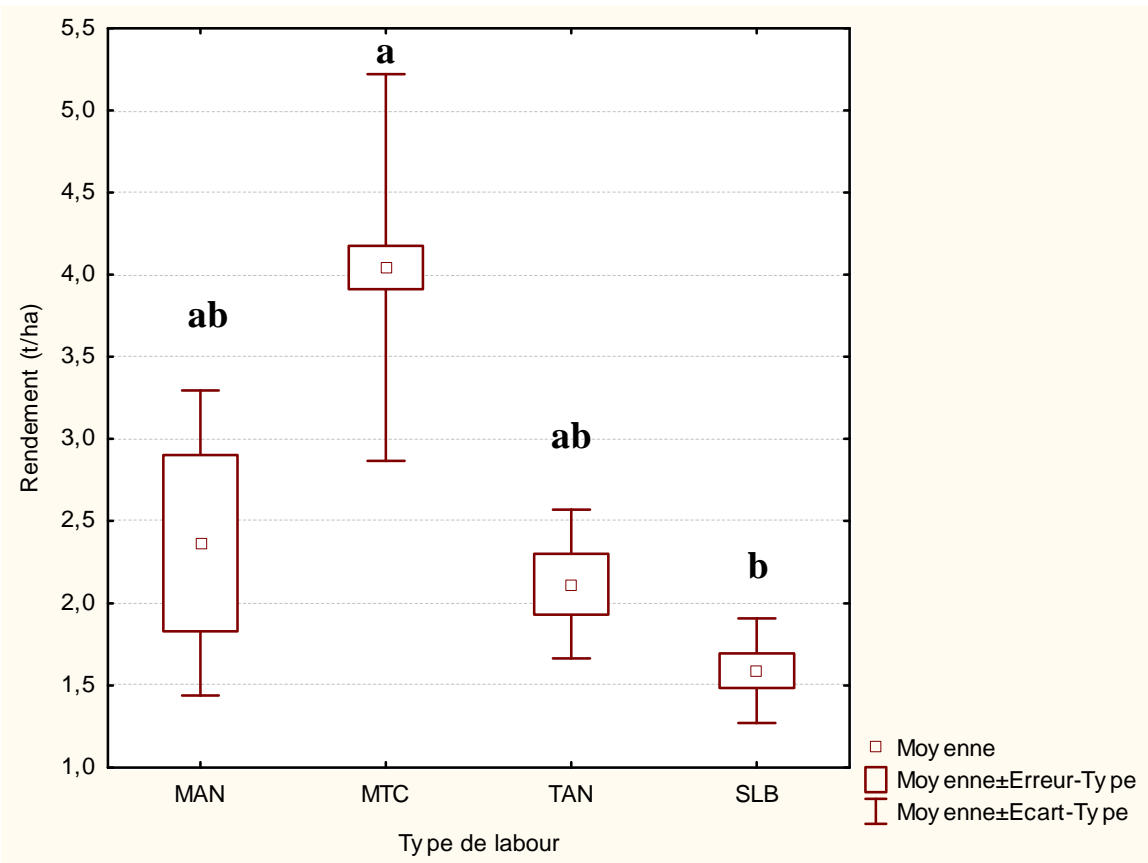

Figure 2 : Comparaison des rendements moyens en fonction des types de labours. 


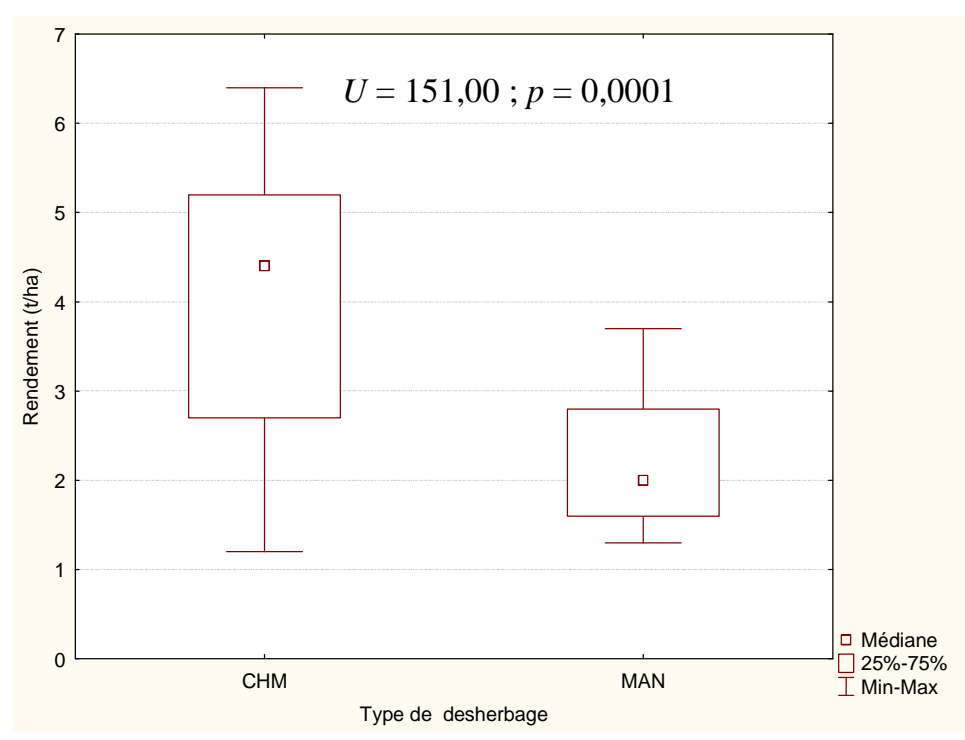

Figure 3 : Comparaison des rendements moyens en fonction des types de désherbages.

\section{DISCUSSION}

\section{Composition floristique}

Les principales familles botaniques observées sont les Cyperaceae et les Poaceae. Le coefficient de similitude entre les deux localités est de $60,87 \%$. Nos résultats sont conformes à ceux de Boraud (2010) effectués sur deux stations voisines au sud de la Côte d'Ivoire en région forestière avec un coefficient de similitude de 57,14\%. Cette forte homogénéité floristique pourrait s'expliquer par le fait que les deux localités explorées sont contiguës géographiquement avec la même végétation et les mêmes pratiques agricoles donc elles renferment un cortège floristique de base.

\section{Impact des pratiques culturales sur le rendement du riz}

Les techniques culturales influencent fortement sur le rendement de la culture du riz. En effet, dans ce travail, les riziculteurs qui n'appliquent aucune technique de labour ont eu le plus faible rendement moyen avec 1,6 t/ha alors que ceux qui pratiquent le labour motorisé ont fait un très bon rendement moyen 4 t/ha. Le labour profond réduit la nuisibilité des adventices en début du cycle cultural surtout lorsqu'il est suivi d'un désherbage chimique. Cependant, le labour motorisé nous offre de bons rendements car il permet de faire des labours profonds.

Les pratiques culturales observées dans cette étude nous ont permis de dire que la riziculture irriguée offre un rendement moyen avec 4,7 t/ha meilleur que 2,4 t/ha dans la riziculture inondée. Cette différence significative de rendement pourrait s'expliquer par la facilité de réaliser la correction d'eau qui consiste à apporter la quantité journalière nécessaire aux besoins physiologiques de la plante et l'utilisation de variétés améliorées dans le système irrigué. Aussi, la bonne gestion de l'eau favorise un bon tallage des plants de riz et par conséquent une bonne production. En plus, les producteurs ont la possibilité de pratiquer plus d'un cycle de culture par campagne selon les zones dans le système irrigué. En outre, le type de labour adopté par les paysans a eu un effet sur leur production. 
Le non labour a un léger avantage de protéger le sol contre l'érosion et conserver l'humidité mais cette pratique offre un habitat très favorable aux mauvaises herbes. Le labour motorisé permet d'enterrer les semences des mauvaises herbes à plus de 20 $\mathrm{cm}$ dans le sol et de ramener à la surface du sol les rhizomes qui sont détruits par un ensoleillement prolongé.

A cela, il faut souligner que le désherbage chimique bien appliqué offre de meilleur rendement moyen de 4,7 t/ha contre 2 t/ha pour le désherbage manuel uniquement. Il donne l'avantage de faire l'application de l'herbicide sur une surface d'un hectare en une journée par personne (Marnotte, 1995). Le désherbage manuel est très pénible pour les grandes surfaces mais efficace sur les petites. Le désherbage chimique a des inconvénients pour l'environnement lorsque les produits phytosanitaires utilisés ne répondent pas aux normes en vigueur.

\section{Conclusion}

Cette étude a permis de recenser 16 espèces végétales réparties en 14 genres appartenant à 10 familles botaniques sur l'ensemble des zones d'étude. Cette flore adventice a été établie pour chaque localité comme suit :

- Bounguè, 8 espèces, 5 genres appartenant à 4 familles botaniques ;

- Raviart, 15 espèces réparties en 14 genres appartenant à 9 familles botaniques.

La localité de culture et le type de labour influencent de façon significative la production du riz. Le labour motorisé pratiqué à Bounguè offre un rendement de $4 \mathrm{t} /$ ha contre 2,4 t/ha à Raviart. Toutefois, les pratiques culturales dans une meilleure adéquation doivent offrir une solution efficace dans la lutte contre les adventices. Ceci est d'autant plus vrai que l'objectif est de trouver une combinaison de méthodes de luttes efficaces, économiquement attractives et sans danger pour l'environnement.

Pour faire face aux prochains défis en matière d'alimentation et de protection de l'environnement, de nombreuses voies ont été proposées ces dernières décennies. Dans le cas de la gestion des adventices, il s'agit de limiter les populations d'adventices à des niveaux acceptables en réduisant l'utilisation des herbicides pour mieux préserver l'environnement.

Il est nécessaire d'entreprendre des recherches et des études pour trouver un panel large de techniques agronomiques bénéfiques aux agriculteurs. Alors, il serait très intéressant de confronter des chercheurs et agriculteurs afin de mener à bien divers projets d'expérimentation en vue de trouver des pratiques alternativement innovantes et durables.

\section{REFERENCES}

Bachelet R. 2012. Recueil, analyse \& traitement de données : Le questionnaire. Présentation, École Centrale de Lille Villeneuve d'Ascq. Disponible sur http://rb.eclille.fr/l/ Analyse_de_donnees/Methodologie_C onception_et_administration_de_questi onnaires.pdf (Consulté le 14/05/2014).

Benzecri JB. 1964. Sur l'analyse factorielle des proximités. Publication de Statistiques de l'Université de Paris 13, France, 235-282.

Benzecri JB. 1973. L'Analyse des Données I : La Taxinomie. Dunod Edition: Paris, France ; 615 .

Bouet A, N'cho AL, Kéli ZJ, Yoboué N, Yayha CM, N'Guessan P. 2005. Bien cultiver le riz irrigué et pluvial en Côte d'Ivoire. CNRA (Edition 2004), août 2005, fiche technique, $4 \mathrm{p}$.

Boraud NKM. 2010. Contribution à l'étude de la simulation d'un impact agroécologique de cultures transgéniques sur la flore adventice de quelques localités de la Côte d'Ivoire. Thèse de Doctorat d'Etat, à l'Université de Cocody-Abidjan, Côte d'Ivoire, 209 p.

Cibois P. 2003. Les méthodes d'analyse d'enquêtes. Rapport, Université de 
Versailles - St-Quentin en Yvelines. Disponible sur : cibois.pagespersoorange.fr/PhCiboisMethAnaEnq.pdf

Djato KK, Dugue P, Pecqueur B. 2006. Interventions publiques pour le développement d'un système agroalimentaire localisé dans le secteur de la production rizicole irriguée en côte d'ivoire. Le Monde en développement. http://www.cairn. info/revue, 101-118

FAO. 2008. Bilan alimentaire de la Côte d'Ivoire. Direction des statistiques, de la documentation et de l'informatique. Ministère de l'agriculture en Côte d'Ivoire, 1-84.

Fenelon JP. 1999. Qu'est-ce que l'Analyse des Données? SEISAM Collection Lefonen : Paris ; France; 311.

Kouamé KF. 2014. Biologie et écologie des adventices majeures de la riziculture dans la région du Bélier au Centre de la Côte d'Ivoire. Thèse unique de Doctorat à l'Université FHB Abidjan, $157 \mathrm{p}$.
Marnotte P. 1995. Utilisation des herbicides: contraintes et perspectives. Agriculture et Développement $\mathrm{N}^{\circ}$, Septembre 1995, 12-21.

Oerke EC, Dehne HW. 2004. Safeguarding production-losses in major crops and the role of crop protection. Crop. Prod., 23: 275-285.

Rodenburg J, Johnson DE. 2009. Weed Management in Rice-Based Cropping Systems in Africa. Advances in Agronomy Elsevier Inc., 103 : 150-201.

Sangaré A, Koffi E, Akamou F, Fall C. 2009. État des ressources phytogénétiques pour l'alimentation et l'agriculture: second rapport national. Ministère de l'agriculture, République de Côte d'Ivoire, $16 \mathrm{p}$.

Sørensen T. 1948. A method of establishing groups of equal amplitude in plant sociology based on similarity of species content. Det Kongelige Danske Videnskabernes Selskabs Kopenhague, Biologiske Skrifter, 5(4): 1-34. 